\title{
Protokoll der Jahresversammlung der Schweizerischen Entomologischen Gesellschaft vom 1. und 2. März 2019 am Naturmuseum St. Gallen
}

\author{
Matthias Borer ${ }^{1}$ \\ 1 Naturhistorisches Museum Basel, Basel, Switzerland \\ http://zoobank.org/1C6EBA64-FD91-485B-AB4C-440A3001D5F \\ Corresponding author: Matthias Borer (matthias.borer@bs.ch)
}

Received 8 November 2019

Accepted 9 Noveber 2019

Published 14 November 2019

Academic editor:

Thibault Lachat

Die Jahresversammlung der SEG wurde auf Einladung des Naturmuseums St. Gallen am 1. und 2. März 2019 in St. Gallen durchgeführt. Der Freitagnachmittag war, neben einem Einblick in die spannenden und noch immer nicht vollständig verstandenen Zyklen der Lärchenwickler, vor allem Themen der angewandten Entomologie in der Forst- und Landwirtschaft sowie in urbanen Gebieten gewidmet. David Frey (Eidg. Forschungsanstalt WSL, Birmensdorf) eröffnete die Tagung mit einem interessanten Hauptvortrag zum Thema Biodiversität und Ökosystemleistungen in Stadtgärten. Im Rahmen des interdisziplinären Nationalfonds-Projekts „BetterGardens“ wurde der Einfluss von 80 privat bewirtschafteten stadtzürcher Gärten auf die Ökosystemleistungen untersucht. Zusätzlich wurde auch die spannende Wechselwirkung zwischen der sozialen Funktion und Ökologie der Gärten erforscht. Die erhobenen Daten zeigen, dass sich extensiv gepflegte und strukturreiche Gärten sowohl positiv auf verschiedene Ökosystemleistungen auswirken als auch dem Stressabbau der Gärtnerinnen und Gärtner dienen. Fazit: Selbst kleine Grünflächen im urbanen Raum können die Biodiversität und diverse Ökosystemprozesse positiv beeinflussen.

\section{Folgende sieben Vorträge wurden am Freitagnach- mittag gehalten:}

- Biodiversität und Ökosystemleistungen in Stadtgärten David Frey, Eidg. Forschungsanstalt WSL, Birmensdorf

- Do cities homogenize biodiversity?

Dr. Marco Moretti, Swiss Federal Research Institute WSL, Birmensdorf
- Effets de l'augmentation de la pollution lumineuse sur les pollinisateurs et la qualité de leur pollinisation Simone Giavi, Institut d'Écologie et Évolution, Université de Berne

- An overview on bee health: are neonicotinoid pesticides to blame?

Dr. Lars Straub, Institut für Bienengesundheit, Bern

- Crab spiders impact floral-signal evolution indirectly through removal of florivores

Anina Knauer, Agroscope, Zürich

- Entomologische Bewertung von Waldrändern Jürg Schlegel, ZHAW, Wädenswil

- Revival der berühmten Lärchenwickler-Zyklen (Zeiraphera griseana)?

Dr. Beat Wermelinger, Eidg. Forschungsanstalt WSL, Birmensdorf

Im Anschluss an die Vorträge wurde zu einer Besichtigung des didaktischen Bienenzentrums beim Gymnasium Untere Waid eingeladen. Den Teilnehmenden wurden die sehr informativ und äusserst ansprechend gestalteten Stationen im Aussenbereich vorgestellt sowie ein Einblick in den sehr divers ausgestatteten Experimentier- und Schulungsraum gewährt. An dieser Stelle ein herzliches Dankeschön an den Präsidenten und die Mitglieder des Bienenzüchtervereins St. Gallen und Umgebung. 
Der Samstag war freien Themen aus der Faunistik, Taxonomie, Systematik, sowie der Datenerhebung mittels Einbezug der Bevölkerung (Citizen Science) gewidmet.

Prof. Dr. Michael Boppré (Albert-Ludwigs-Universität, Freiburg i. Br.) eröffnete den zweiten Tag mit einem facettenreichen und spannenden Vortrag - einem Einblick in seine jahrzehntelange Erforschung der Bärenspinner in der Neotropis. Die attraktiven Farbmuster sind auch für Nicht-Spezialisten dieser Gruppe sehr überzeugend. Herr Boppré zeigte, dass diese Bärenspinner in verschiedenster Weise, sei es durch ihre Morphologie, Physiologie / Biochemie, Ökologie oder auch ihr Verhalten sehr spannend, divers und daher auch anspruchsvoll zum Erforschen sind. Diese Variabilität ist dann auch für das taxonomische Bearbeiten und die Systematik dieser Gruppe eine grosse Herausforderung. $\mathrm{Zu}$ den biologischen Herausforderungen der untersuchten Organismen kommen leider immer wie mehr komplizierte und aufwändige bürokratische Hindernisse dazu, die das Erforschen von Organismen ganz allgemein extrem erschweren oder sogar verunmöglichen. Der Referent hielt einen äusserst gelungenen und überzeugenden Vortrag - ein Plädoyer für die Grundlagenforschung in der Entomologie.

Folgende zehn Vorträge wurden am Samstag gehalten:

- Bären in der Neotropis: Lust und Frust mit Spinnern (Lep.: Erebidae: Arctiinae)

Prof. Dr. Michael Boppré, Albert-Ludwigs-Universität, Freiburg i.Br.

- Tapinoma magnum, une nouvelle espèce de fourmi en Suisse, invasive et redoutable!

Dr. Anne Freitag, Musée de Zoologie Lausanne \& Dr. Daniel Cherix, Université de Lausanne

- Kontaktzonen und zwischenartlicher Genfluss zwischen drei nahverwandten Arten des Erebia tyndarus Komplexes

Dr. Kay Lucek, Universität Basel

- Regeln für das Züchten von Schmetterlingen Marc de Roche, Swiss Butterfly Breeders, Bern

- Neotropical jumping plant-lice (Hemiptera, Psylloidea) associated with Copaifera and Hymenaea (Leguminosae, Detarioideae, Detarieae)

Dr. Daniel Burckhardt, Naturhistorisches Museum Basel

- Untersuchungen zur Totholzkäferfauna im Kanton St. Gallen

Barbara Huber, Abenis AG, Chur

- Käferendemiten in Österreich - Übersicht und Updates Mag. Gregor Degasperi, Innsbruck, Österreich

- Bürgerbeteiligung und die Dokumentation der Artenvielfalt - Fallstricke und Chancen

Dr. Georg Friebe, Inatura GmbH, Dornbirn
- Klimawandel fördert Ausbreitung von Scutigera coleoptrata

Mag. Dr. Klaus Zimmermann, Inatura GmbH, Dornbirn

- Aedes albopictus in its native range:NGS-based analysis of the population structure of the Asian tiger mosquito Dr. Ann-Christin Honnen, Swiss TPH (Schweizerisches Tropen- und Public Health-Institut), Basel

Die Vortragsreihe am Samstag wurde mit einer Führung in die Sammlung des Naturmuseums St. Gallen abgerundet.

An der Generalversammlung vom Samstagmorgen nahmen 22 Mitglieder teil. Dem Naturmuseum St. Gallen, im speziellen Andreas Kopp und Priska Seri-Jud, sei für die hervorragende Organisation der Jahresversammlung und für das Offerieren der Pausenverpflegungen herzlich gedankt.

\section{Generalversammlung}

\section{Begrüssung}

Der Präsident Hannes Baur eröffnet die Generalversammlung um 10:00 und begrüsst die 22 anwesenden Mitglieder und 8 Gäste.

\section{Protokoll der Generalversammlung 2017 in Bern}

Das Protokoll wird kommentarlos genehmigt.

\section{Bericht des Präsidenten (Hannes Baur)}

\section{Administratives}

Im administrativen Bereich wurden wir 2018 wiederum durch das Centre Suisse de Cartographie de la Faune (CSCF) in Neuchâtel unterstützt. Die SEG ist Mitglied der Plattform Biologie der Akademie der Naturwissenschaften Schweiz (SCNAT) in Bern und wird dort von der Geschäftsleiterin Pia Stieger betreut.

\section{Finanzielle Unterstützung}

Die Gesuche des Präsidenten um finanzielle Unterstützung wurden von den angefragten Institutionen in vollem Umfang bewilligt. Es handelt sich dabei um folgende Beträge: Akademie der Naturwissenschaften Schweiz (SCNAT) in Bern: CHF 7‘500.-, Biedermann-MantelStiftung in Zürich: CHF 6'000.- und Syngenta AG in Basel: CHF 6`000.-. Wir danken allen drei Geldgebern für ihre wichtigen Beiträge.

Jahresversammlung, Generalversammlung und Vorstandssitzungen

Die entomo.ch, die traditionell zweitägige Jahresversammlung der SEG, wurde am Freitag, 2. und Samstag, 3. März 2018 am Naturhistorischen Museum Bern durchgeführt. Dem Museumsteam, insbesondere der Cafeteria, danken wir für die Organisation des 
Anlasses ganz herzlich. Die Generalversammlung fand am Samstag, 3. März 2018 von 10 bis 11 Uhr am gleichen Ort statt. Der Präsident leitete die beiden regulären, halbjährlichen Vorstandssitzungen, welche am Freitag, 2. März und am Mittwoch, 7. November 2018 jeweils am Naturhistorischen Museum in Bern stattfanden.

\section{Website}

Die Website unserer Gesellschaft, u.a. mit Information zur Jahrestagung entomo.ch, war auch dieses Jahr wieder im Portal Naturwissenschaften Schweiz der Akademie der Naturwissenschaften Schweiz (SCNAT) abrufbar. Für die Betreuung danke ich Pascal Blanc (Chefredaktor) sowie Stefan Schmidlin und Mariella Hobi (Support). https://www.naturwissenschaften.ch/organisations/seg

\section{Digitalisierung von Sammlungen}

Die SCNAT hat eine Initiative zur Digitalisierung aller naturwissenschaftlichen Sammlungen der Schweiz gestartet. Grundlage dazu ist ein Bericht, welcher unter der Federführung des neuen Präsidenten der Plattform Biologie, Prof. Dr. Christoph Scheidegger von der WSL Birmensdorf, ausgearbeitet wurde (Swiss Academies of Arts and Sciences 2019). Im Bericht wurde aufgezeigt, wo die Defizite beim Zugang zu den zahlreichen Sammlungen liegen. Ein grosser Teil der über 60 Millionen Objekten ist demnach nur ungenügend erschlossen. Dies gilt insbesondere für die Insekten. Der Präsident hatte im Berichtsjahr als Vertreter der SEG an einem Symposium und einem Workshop zu diesem Thema teilgenommen. Viel Raum hatte es auch an der Sitzung der Plattform Biologie, wo der Präsident ebenfalls teilnahm.

\section{Insektensterben}

Seit der Publikation der sogenannten Krefelder Studie (Hallmann et al. 2017) hat der Rückgang der Insekten unter dem Titel „Insektensterben“ weltweit grosse Beachtung in den Medien gefunden. Der Präsident wurde während der Berichtsperiode verschiedentlich um Stellungnahme aus Sicht unserer Gesellschaft gebeten. Er nahm diesbezüglich auch an einem Podiumsgespräch in Luzern teil. Der SEG-Vorstand wird an einer der kommenden Sitzungen das Thema aufgreifen.

\section{Insekt des Jahres}

In Zusammenarbeit mit Entomologen aus Deutschland und Österreich wurde für das Jahr 2019 die Rostrote Mauerbiene (Osmia bicornis) zum Insekt des Jahres bestimmt. Dazu wurde wiederum ein Flyer publiziert, welcher über die SEG bezogen werden kann.

Im Namen des Vorstandes und der Mitglieder der Schweizerischen Entomologischen Gesellschaft danke ich allen genannten Institutionen, Gremien und Personen nochmals ausdrücklich für ihre Unterstützung und ihren Einsatz!
Bericht des Bibliothekars und der Redaktoren (Alpine Entomology und Fauna Helvetica)

Bericht des Bibliothekars (Dr. P. Jeanneret)

En 2018, quelques demandes d'articles ont été enregistrées. J'ai alors fait référence à la version électronique d'Alpine Entomology. Quelques demandes concernant les échanges de l'ex-bulletin avec des publications étrangères ont été formulées. J'ai alors à nouveau référé à la version électronique d'Alpine Entomology et j'ai annoncé que la bibliothèque de l'ETH ne reprenait plus de version papier de publications pour la société.

Bericht des Redaktors der Fauna Helvetica (PD Dr. D. Burckhardt)

Im Berichtsjahr wurden die Bände 29 und 30 publiziert. Beide sind überarbeitete Neuauflagen von Werken, die seit einiger Zeit vergriffen sind. Bei Band 29 handelt es sich um den ersten Teil der Apidae, der 1996 als letzter Band der Insecta Helvetica publiziert wurde. Neben der Einführung in die Apidae werden vor allem die Bombus-Arten abgehandelt. Band 30 ist eine Neubearbeitung der Fische. Im Weiteren ist Band 32 über die Amphipoda im Druck. Der Band über die Vespidae befindet sich im Stadium der Drucklegung und sollte ebenfalls bald publiziert sein. An weiteren Bänden wird gearbeitet.

Wie immer klappte die Zusammenarbeit mit dem CSCF ausgezeichnet. Dafür möchte ich dem Leiter Dr. Y. Gonseth und seinen Mitarbeiterinnen und Mitarbeitern ganz herzlich danken.

Bericht des Chefredaktors von Alpine Entomology (Dr. T. Lachat)

The second issue of Alpine Entomology arrived in time for Christmas 2018. This issue includes 14 articles on 160 pages: eleven research articles, one book review, one editorial and one meeting report of the Swiss Entomological Society. Nine of eleven research articles have been published in English. This shows that our journal is gaining in popularity among entomologists worldwide.

The editorial board is now composed of twenty specialists from all fields of Entomology and from a variety of different countries. Summing up the review process for the second issue, six manuscripts have been rejected, however resubmission was encouraged for half of them.

We think that the transition from the former "Mitteilungen der Schweizerischen Entomologischen Gesellschaft" has been achieved and we can consider the last two years as a success. The excellent support of Pensoft plays a major role in this endeavor, together with the very active support of our editorial board and reviewers. Our next aim in the coming years is to gain recognition for our journal by the Science Citation Index Expanded (SCIE) in order to be considered as a journal with impact factor. 


\section{Bericht der Quästorin und der Rechnungsrevisoren}

Bericht der Quästorin (E. Leonetti)

Frau Emanuela Leonetti legt den Anwesenden die Jahresrechnung 2018 vor. Daraus sind folgende Zahlen entnommen:

\begin{tabular}{|c|c|c|}
\hline Positionen/Objets & $\begin{array}{l}\text { Ausgaben/ } \\
\text { Dépenses }\end{array}$ & $\begin{array}{l}\text { Einnahmen/ } \\
\text { Revenus }\end{array}$ \\
\hline \multicolumn{3}{|l|}{$\begin{array}{l}\text { Publikationskosten / Charges de } \\
\text { publications: }\end{array}$} \\
\hline Publications AE - impression, rédaction & 7’059.89 & \\
\hline Pensoft: Website, Open access & $17 ’ 376.75$ & \\
\hline Verwaltung / Administration & $5 ‘ 287.25$ & \\
\hline Beitrag sc|nat / Cotisation sc|nat & $1^{\prime} 645.00$ & \\
\hline $\begin{array}{l}\text { Arbeitsgruppenförderung / Soutien } \\
\text { groupes de travail }\end{array}$ & $2 ‘ 187.75$ & \\
\hline Verkauf Mitteilungen / Ventes bulletins & & $1 ‘ 307.05$ \\
\hline Mitgliederbeiträge / Cotisations & & $15 ‘ 401.57$ \\
\hline \multicolumn{3}{|l|}{ Beiträge / Subventions } \\
\hline sc|nat & & $12^{\prime} 187.75$ \\
\hline Syngenta & & 6.000 .00 \\
\hline Biedermann - Mante I- Stiftung & & $6 ، 000.00$ \\
\hline $\begin{array}{l}\text { Spenden und sonstige Einnahmen / Dons } \\
\text { et autres produits }\end{array}$ & & 90.00 \\
\hline Zinsen / Intérêts & 135.60 & \\
\hline Total / Totaux & $33 \prime 692.24$ & $40 \prime 986.37$ \\
\hline \multirow[t]{2}{*}{ Jahresgewinn / Bénéfice annuel 2018} & 7‘294.13 & \\
\hline & $40 ’ 986.37$ & $40^{\prime} 986.37$ \\
\hline
\end{tabular}

Die Erfolgsrechnung für das Jahr 2018 schloss mit einem Gewinn von CHF 7'294.13 (Vermögen CHF 102'834.66).

Bericht der Rechnungsrevisoren (Dr. M. Sartori und Andreas Sanchez)

En tant qu'organe de contrôle de la société Entomologique Suisse, nous avons vérifié les comptes de l'exercice 2018. Nous avons pu constater que:

- le bilan d'ouverture, le bilan de clôture et le compte d'exploitation correspondent à la comptabilité

- les justificatifs sont conformes, exacts et correspondent à l'activité habituelle de la SES

- la fortune de la société correspond aux comptes à l'actif du bilan (liquidité + transitoires).

Pour sa part, la caissière assure que la tenue de la comptabilité a été faite, dans les limites de ses compétences, avec exactitude et selon les principes formulés dans le Code des Obligations (CO 959 t 662a/2).

C'est pourquoi nous prions l'assemblée d'accepter les comptes de l'exercice 2017 et de donner décharge à la caissière.

Der Quästorin wird einstimmig Decharge erteilt.

\section{Budget und Mitgliederbeitrag}

Budget 2019

Der Präsident stellt das Budget für 2019 vor, das einen Gewinn von CHF 1'400.- vorsieht.
Mitgliederbeitrag 2019

Die Beiträge bleiben gleich: Mitglieder in der Schweiz CHF 60.-, Studierende oder sich anderweitig in Ausbildung befindende Mitglieder zahlen während drei Jahren nur die Hälfte (CHF 30.-) des ordentlichen Beitrages. Mitgliedern im Ausland, die die gedruckte Version von Alpine Entomology wünschen, werden zusätzlich $\mathrm{CHF}$ 15.- Versandkosten verrechnet.

Das Budget und der Mitgliederbeitrag werden einstimmig und ohne Enthaltung genehmigt.

\section{Prix Moulines 2019}

Der Präsident gibt den Gewinner, Dr. Beat Wermelinger, bekannt. Herr Wermelinger, ein enthusiastischer Makrofotograf, wird für sein Buch «Insekten im Wald. Vielfalt, Funktion und Bedeutung», das an ein breites Publikum gerichtet, aber dennoch ein Fachbuch ist, ausgezeichnet.

Wermelinger B., 2017: Insekten im Wald. Vielfalt, Funktionen und Bedeutung. Birmensdorf, Eidg. Forschungsanstalt WSL; Bern, Stuttgart, Wien, Haupt Verlag. 367 S.

\section{Varia}

Der Präsident gratuliert dem Mitglied Dr. h.c. Armin Coray im Namen der SEG zu seinem Ehrendoktortitel, der ihm im November 2018 von der Universität Basel verliehen wurde.

\section{Jahresversammlung 2020}

Die nächste Jahresversammlung, entomo.ch 2020, findet am 6. und 7. März 2020 am Naturhistorischen Museum in Basel statt.

Ende der Generalversammlung um 10:50 Uhr.

Basel, im November 2019, der Sekretär Matthias Borer

\section{Referenzen}

Hallmann CA, Sorg M, Jongejans E, Siepel H, Hofland N, Schwan H, Stenmans W, Müller A, Sumser H, Hörren T, Goulson D, Kroon H de (2017) More than 75 percent decline over 27 years in total flying insect biomass in protected areas. PLOS ONE 12: e 0185809. https:// doi.org/10.1371/journal.pone.0185809

Swiss Academies of Arts and Sciences (2019) National significance of natural history collections in Switzerland. Swiss Academies Reports 14(2): 36 p. https://naturwissenschaften.ch/service/publications/109910-nationale-bedeutung-naturwissenschaftlicher-sammlungen-der-schweiz 